\title{
Quantitative Bioreactor Monitoring of Intracellular Bacterial Metabolites in Clostridium autoethanogenum Using Liquid Chromatography-Isotope Dilution Mass Spectrometry
}

Laudina Safo," Salah Abdelrazig," Alexander Grosse-Honebrink, Thomas Millat, Anne M. Henstra, Rupert Norman, Neil R. Thomas, Klaus Winzer, Nigel P. Minton, Dong-Hyun Kim, and David A. Barrett*

Cite This: ACS Omega 2021, 6, 13518-13526

Read Online

ABSTRACT: We report a liquid chromatography-isotope dilution mass spectrometry method for the simultaneous quantification of 131 intracellular bacterial metabolites of Clostridium autoethanogenum. A comprehensive mixture of uniformly ${ }^{13} \mathrm{C}$-labeled internal standards (U- ${ }^{13} \mathrm{C}$ IS) was biosynthesized from the closely related bacterium Clostridium pasteurianum using $4 \%{ }^{13} \mathrm{C}$-glucose as a carbon source. The $\mathrm{U}-{ }^{13} \mathrm{C}$ IS mixture combined with ${ }^{12} \mathrm{C}$ authentic standards was used to validate the linearity, precision, accuracy, repeatability, limits of detection, and quantification for each metabolite. A robust-fitting algorithm was employed to reduce the weight of the outliers on the quantification data. The metabolite calibration curves were linear with $R^{2} \geq 0.99$, limits of detection were $\leq 1.0 \mu \mathrm{M}$, limits of
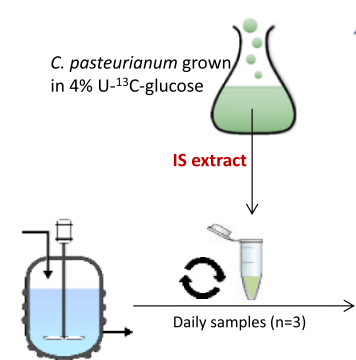

Bioreactor under $\mathrm{pH}$ Extracted bacterial samples modulation quantification were $\leq 10 \mu \mathrm{M}$, and precision/accuracy was within RSDs of $15 \%$ for all metabolites. The method was subsequently applied for the daily monitoring of the intracellular metabolites of $C$. autoethanogenum during a $\mathrm{CO}$ gas fermentation over 40 days as part of a study to optimize biofuel production. The concentrations of the metabolites were estimated at steady states of different $\mathrm{pH}$ levels using the robust-fitting mathematical approach, and we demonstrate improved accuracy of results compared to conventional regression. Metabolic pathway analysis showed that reactions of the incomplete (branched) tricarboxylic acid "cycle" were the most affected pathways associated with the $\mathrm{pH}$ shift in the bioreactor fermentation of C. autoethanogenum and the concomitant changes in ethanol production.

\section{INTRODUCTION}

Absolute quantification of intracellular metabolite concentrations in microorganisms is a crucial step in providing precise information for accurate quantitative modeling of the intracellular processes in systems biology and metabolic engineering. Untargeted metabolomics, using liquid chromatographyhigh-resolution mass spectrometry (LC-HRMS), aims to provide an unbiased picture of the entire metabolite inventory of the cells, ${ }^{1}$ but this approach often does not provide absolute concentrations and can deliver ambiguous metabolite identification. Hence, the inclusion of metabolite data from such untargeted methods can result in misleading or unreliable metabolic models. ${ }^{2}$ Absolute quantification using LC-isotope dilution MS (LC-IDMS) with synthesized isotopic internal standards (ISs) can correct for the majority of the LC-MS bias and hence is regarded as the "gold standard" for the determination of metabolite concentrations. ${ }^{3}$ These targeted LC-HRMS methods can reduce the uncertainty in data and generate a high level of confidence in metabolite identities and measured concentrations. ${ }^{4}$ However, these advantages come with a requirement for a substantial additional effort in method validation and optimization, particularly with respect to the use of multiple IS and data handling.

To generate valid models of bacterial metabolism, it is increasingly necessary to expand measurements to cover most of the key central metabolic pathways, requiring over 100 metabolites to be quantified from a single sample. Crucially, many of these metabolic compounds, including key intermediates, are not readily available as ${ }^{13} \mathrm{C}$ isotopically labeled forms, thus limiting the scope of applied LC-IDMS methods. To overcome this bottleneck, a promising and convenient

Received: November 19, 2020

Accepted: February 3, 2021

Published: May 20, 2021 
alternative is the biosynthesis of multiple ${ }^{13} \mathrm{C}$-labeled chemical IS using microorganisms such as Escherichia coli, ${ }^{5}$ spirulina, ${ }^{6}$ or yeast ${ }^{7}$ grown on isotopically labeled substrates. Recently, we reported the use of commercially available uniformly ${ }^{13} \mathrm{C}$ labeled (U- ${ }^{13} \mathrm{C}$ ) spirulina (Arthrospira platensis) as an accessible source of $\mathrm{U}^{13} \mathrm{C}$ IS for the absolute quantification and monitoring of intracellular metabolites during bacterial gas fermentation. ${ }^{6}$ Our current steady-state, continuous culture bioreactor studies of Clostridium autoethanogenum required the analysis of a wider set of central metabolites and the need for highly robust analytical data to support metabolic modeling. Based on these needs, we have developed a new approach to (a) cover a more comprehensive range of metabolites and (b) explore the use of error correction algorithms to improve data quality.

The focus of our work is acetogen $C$. autoethanogenum and its potential use for the production of biofuel additives such as ethanol and 2,3-butanediol synthetic products such as isopropanol through carbon monoxide/carbon dioxide ( $\mathrm{CO} /$ $\mathrm{CO}_{2}$ ) fixation. ${ }^{8-10}$ The endogenous biosynthesis of these synthetic products by $C$. autoethanogenum is minimal; hence, metabolic engineering and optimization of the growth conditions are required to maximize productivity. Our method has been specifically developed to understand the metabolic changes associated with the conversion of acetate to ethanol by C. autoethanogenum at a low $\mathrm{pH}$ of the growth media. ${ }^{11-13}$ The biosynthetic generation of the required $\mathrm{U}^{13} \mathrm{C}-\mathrm{IS}$ from $C$. autoethanogenum is difficult due to the challenges associated with the use of ${ }^{13} \mathrm{C}$-labeled gases such as $\mathrm{CO}_{2}$ and $\mathrm{CO}$ as carbon sources and the low biomass obtained from this organism compared with other clostridial species using ${ }^{13} \mathrm{C}$ sugar substrates. Therefore, we evaluated the use of a closely related organism Clostridium pasteurianum for the biological synthesis of the required multiple $\mathrm{U}_{-}{ }^{13} \mathrm{C}$-IS.

The measured concentration of metabolites over the course of a bioreactor run (often several weeks) is subject to biological and analytical variability, occasionally resulting in outliers in the data sets. ${ }^{14}$ Such outliers have the potential to significantly distort the estimated concentrations of bioreactor metabolites at a steady state and are thus a serious concern for experimental design and computational modeling. ${ }^{15}$ Robust regression has been used in clinical metabolomics to uncover metabolic rewiring in disease states using GC-MS. ${ }^{16}$ However, it has not been applied in bacterial metabolomics and it has the potential to reduce the impact of outliers in the analysis and to improve the processing of large and complex data sets from quantitative metabolomics.

Here, we demonstrate a validated LC-IDMS method using multiple $\mathrm{U}_{-}{ }^{13} \mathrm{C}-\mathrm{IS}$ generated from $C$. pasteurianum for the monitoring of 131 metabolites during the $\mathrm{CO}$ gas-based fermentation of $C$. autoethanogenum over 40 days. Additionally, we investigate an automated workflow that combines nonlinear least-square regression with robust statistics to improve the accurate estimation of metabolite concentrations for incorporation into computational modeling.

\section{EXPERIMENTAL SECTION}

Chemicals. Uniformly ${ }^{13} \mathrm{C}$-labeled glucose (U- ${ }^{13} \mathrm{C}, 97 \%$ ) was purchased from CK Isotopes Laboratory (Ibstock, UK). C. autoethanogenum (DSM 10061) and C. pasteurianum (DSM 525) were purchased from the Leibniz Institute DSMZGerman Collection of Microorganisms and Cell Cultures $\mathrm{GmbH}$ (Braunschweig, Germany). HPLC grade ammonium carbonate and all other analytical standards were purchased from Sigma-Aldrich (Gillingham, UK). MS grade acetonitrile and methanol were purchased from VWR (Lutterworth, UK). Individual stock solutions of $100 \mathrm{mM}$ authentic standards of $131{ }^{12} \mathrm{C}$ metabolites (Table $\mathrm{S} 1$ ) from selected metabolic pathways of interest of $C$. autoethanogenum were prepared in $50 \%(\mathrm{v} / \mathrm{v})$ methanol/water and diluted as appropriate for LCIDMS analysis.

LC-IDMS Analysis. LC-IDMS was performed on a ZIC$p$ HILIC column $(4.6 \times 150 \mathrm{~mm}, 5 \mu \mathrm{m}$ particle size, Merck SeQuant, Watford, UK) using Dionex UHPLC coupled to a QExactive Plus hybrid quadrupole-Orbitrap mass spectrometer (Thermo Fisher Scientific, USA). A full description of the LC-IDMS method, developed from our previous publication, ${ }^{6}$ is given in the Supporting Information.

Biosynthesis and Extraction of $\mathrm{U}^{13} \mathrm{C}$-IS from $C$. pasteurianum Grown on $\mathrm{U}^{13} \mathrm{C}-\mathrm{Glucose}$. C. pasteurianum (DSM 525) was used to generate multiple U- ${ }^{13} \mathrm{C}$-IS for LCIDMS. The organism was activated on a clostridial basal medium (CBM) agar, as detailed elsewhere. ${ }^{17}$ Frozen cells $\left(-80{ }^{\circ} \mathrm{C}\right)$ were streaked out on a prewarmed $\left(37^{\circ} \mathrm{C}\right)$, reduced $\mathrm{CBM}$ agar plate in an anaerobic cabinet (Don Whitley Scientific, UK) containing an 80:10:10 $\mathrm{N}_{2} / \mathrm{CO}_{2} / \mathrm{H}_{2}$ atmosphere and incubated at $37{ }^{\circ} \mathrm{C}$ for $48 \mathrm{~h}$. Actively growing cells scraped from this plate were then used as an inoculum in a modified liquid Biebl medium ${ }^{18}$ which was then incubated for further $24 \mathrm{~h}$ under the same conditions. Details of the modified Biebl medium are given in the Supporting Information. The growth of $C$. pasteurianum was monitored by measuring the optical density at $600 \mathrm{~nm}\left(\mathrm{OD}_{600}\right)$ over $24 \mathrm{~h}$.

The cells of $C$. pasteurianum were harvested by pipetting 1 $\mathrm{mL}$ of aliquots of the whole culture immediately into precooled tubes on ice and centrifuged $(10,000 g, 2 \mathrm{~min})$. The supernatants were removed, and the multiple U- ${ }^{13} \mathrm{C}-\mathrm{IS}$ from the cell pellets were extracted using $1 \mathrm{~mL}$ of methanol $\left(-20{ }^{\circ} \mathrm{C}\right)$ and vortexed for $1 \mathrm{~min}$. The resuspended cells were lysed by freezing in liquid nitrogen, thawed on ice, and vortexed for $30 \mathrm{~s}$. The freeze-thaw sequence was repeated three times. The final extract was centrifuged $(10,000 \mathrm{~g}, 5 \mathrm{~min})$ to remove cell debris, and the supernatant was removed and stored at $-80{ }^{\circ} \mathrm{C}$ to be used as a $\mathrm{U}^{13} \mathrm{C}$-IS mixture for $\mathrm{LC}-$ IDMS (Table S1).

Validation of LC-IDMS Using U- ${ }^{13} \mathrm{C}$-IS Extracted from C. pasteurianum. Data analysis was performed using TraceFinder 3.1 (Thermo Fisher Scientific, Hemel Hempstead, $\mathrm{UK})$. The retention time (RT) and accurate mass $(<5 \mathrm{ppm})$ were used to assign the metabolite/IS peaks. ${ }^{12} \mathrm{C}$ metabolite calibration standards ( 13 calibration points, 131 standards) in the concentration range of $1 \mathrm{nM}$ to $200 \mu \mathrm{M}$ were prepared in $50 \% \mathrm{v} / \mathrm{v} \mathrm{methanol/water} \mathrm{from} \mathrm{each} \mathrm{standard} \mathrm{mixture,} \mathrm{spiked}$ with $1: 1 \mathrm{v} / \mathrm{v} \mathrm{U}-{ }^{13} \mathrm{C}$ extract of C. pasteurianum, and analyzed with LC-IDMS $(n=6)$. The limit of detection (LOD), limit of quantification (LOQ), linearity using coefficient of determination $\left(R^{2}\right)$, precision, accuracy, and repeatability for each standard were used to assess the validity of the LCIDMS based on the acceptance criteria for quantitative LCMS/MS assays in the FDA guidelines for bioanalysis. ${ }^{19}$

Cultivation, Harvest, and Extraction of C. autoethanogenum during a pH-Shift Modulation. C. autoethanogenum (DSM 10061) cells were revived and grown on a continuous $\mathrm{CO}$-fed bioreactor using the conditions and setup stated elsewhere. ${ }^{6}$ The bioreactor was inoculated from the PETC-2-(N-morpholino)ethanesulfonic acid (MES) growth 
medium $^{20}$ with a $\mathrm{CO}$ starter culture. $\mathrm{pH}$ conditions of the culture medium were adjusted throughout the bioreactor run: pH 5.0 at $15 \mathrm{~mL} / \mathrm{min} \mathrm{CO}$ (day $1-11$ ), pH 5.0 at $27 \mathrm{~mL} / \mathrm{min}$ $\mathrm{CO}$ (day 12-18), $\mathrm{pH} 4.7$ at $27 \mathrm{~mL} / \mathrm{min} \mathrm{CO}$ (day 19-21), $\mathrm{pH}$ 4.5 at $27 \mathrm{~mL} / \mathrm{min} \mathrm{CO}$ (day 22-26), and $\mathrm{pH} 5.0$ at $27 \mathrm{~mL} / \mathrm{min}$ CO (day 27-40). The total gas flow was kept constant at 100 $\mathrm{mL} / \mathrm{min}$ by balancing the $\mathrm{N}_{2}$ flow. The production of acetate, ethanol, and 2,3 butanediol throughout the 40 days of the bioreactor run was monitored using an HPLC system, as previously described. ${ }^{6}$

Bioreactor samples of $C$. autoethanogenum were collected at $37{ }^{\circ} \mathrm{C}$ when the optical density at $600 \mathrm{~nm}\left(\mathrm{OD}_{600}\right)$ reached 4.00. Daily samples of $1 \mathrm{~mL}(n=3)$ were collected on ice throughout the bioreactor run (40 days) and subsequently centrifuged $\left(4^{\circ} \mathrm{C}, 10,000 \mathrm{~g}\right)$, and following the removal of the supernatants, the cell pellets were snap-frozen in liquid nitrogen and stored at $-80{ }^{\circ} \mathrm{C}$. The intracellular metabolites of $C$. autoethanogenum were extracted from the cell pellets using the freeze-thaw approach described above with $2: 1: 1$ chloroform/methanol/water containing the $1: 1 \mathrm{v} / \mathrm{v} \quad \mathrm{U}_{-}{ }^{13} \mathrm{C}$ extract of C. pasteurianum (U- ${ }^{13} \mathrm{C}-\mathrm{IS}$ ). $50 \mu \mathrm{L}$ of the aqueous supernatants was analyzed with calibration standards in a single analytical LC-IDMS run. Steady states were estimated by the use of robust regression to estimate average metabolite concentrations when the growth conditions remained constant for a period of days.

Robust Regression for the Estimation of Metabolite Concentrations at Different Steady States. An automated workflow that combines nonlinear least-square regression and robust statistics was implemented using Matlab 2019a (MathWorks, Natick, USA) to estimate the intracellular metabolite concentrations at different steady states. ${ }^{15,16}$ Initially, the unweighted data are used to estimate a first set of parameters for the chosen fit function. Then, a parameter $r$ is calculated for each data point based on the residuals (resid) from the previous iteration, an estimate of the standard deviation $s$, leverage values $h$, and a tuning constant (tune)

$$
r=\frac{\text { resid }}{\text { tune } \cdot s \cdot \sqrt{1-h}}
$$

Thus, the parameter $r$ in (1) combines information about the distance of a data point from the found fit, its influence on the regression result, and the width of the distribution of the data point around it. The width of the distribution is calculated from the median absolute deviation of the residuals from their median (MAD)

$$
s=\frac{\mathrm{MAD}}{0.6745}
$$

The constant denominator in (2) is chosen to ensure an unbiased estimate for the normal distribution. Note that the tuning constant is specific to the chosen weighing function and determines the down-weight assigned to large residuals (default values were applied here). Crucial to this approach is the choice of the weighing function $w$. This function is supposed to follow the inherent distribution of the experimental data around the fit function. Consequently, it has a significant effect on the identification of outliers and thus the results of the regression analysis. For this analysis, we assumed that the data points are normally distributed and employed the bisquare function (3)

$$
w= \begin{cases}\left(1-r^{2}\right)^{2} & \text { if } \operatorname{abs}(r)<1 \\ 0 & \text { else }\end{cases}
$$

which counteracts the quadratic impact of residuals obeying a normal distribution on parameter estimation. As defined in (3), the weights decrease with the increasing parameter $r$ (1) and thus reduce the effect of data points with larger residuals on the regression analysis. If the parameter $r$ exceeds \pm 1 , a weight of zero is assigned, which will effectively remove the corresponding data point from the next regression run. This procedure is repeated until the sum of weighted least squares is minimal. ${ }^{21,22}$ Note that the computed confidence interval represents an approximation as no strict derivation exists in contrast to the standard least-square regression approach.

Following the determination of average metabolite concentrations, $p$ values were computed using a two-sided Kolmogorov-Smirnov test to determine whether the change in the metabolite concentrations between any two different steady states is significant or not. To our best knowledge, this approach has not been used in metabolite analysis previously.

\section{RESULTS AND DISCUSSION}

Optimization of the Growth of C. pasteurianum on $\mathrm{U}-{ }^{13} \mathrm{C}-$ Glucose for the Biosynthesis of $\mathrm{U}-{ }^{13} \mathrm{C}-\mathrm{IS}$. The uniformly labeled $C$. pasteurianum biomass was required for the extraction of ${ }^{13} \mathrm{C}$ metabolites. For this strain, DSM 525 was grown on a modified Biebl medium which, in addition to glucose, contained no other carbon compounds other than $\mathrm{CaCO}_{3}(250 \mathrm{~g} / \mathrm{L})$ as a buffering agent and very low concentrations of biotin $(25 \mu \mathrm{g} / \mathrm{L}$; see the Supporting Information for the exact composition). The optimal growth of $C$. pasteurianum was supported by the presence of biotin (25 $\mu \mathrm{g} / \mathrm{L})$ with $\mathrm{CaCO}_{3}(250 \mathrm{~g} / \mathrm{L})$ compared to the use of the MES buffer (Figure 1a). The MES buffer was initially used because of our concerns regarding potential ${ }^{12} \mathrm{C}$ carbon contamination due to $\mathrm{CO}_{2}$ derived from $\mathrm{CaCO}_{3}$. However, the growth with the MES buffer was much poorer than with $\mathrm{CaCO}_{3}$. Hence, the potential of $\mathrm{Ca}^{12} \mathrm{CO}_{3}$ (used as a $\mathrm{pH}$ regulator) to serve as an unwanted carbon source by $C$. pasteurianum was also investigated. $\mathrm{Ca}^{13} \mathrm{CO}_{3}$ addition, with ${ }^{12} \mathrm{C}$ glucose, in the culture medium showed no ${ }^{13} \mathrm{C}$-labeled metabolites and confirmed that $\mathrm{CaCO}_{3}$ was not a carbon source. Hence, $\mathrm{Ca}^{12} \mathrm{CO}_{3}$ can be used in the production of the ${ }^{13} \mathrm{C}$-labeled biomass without risking ${ }^{12} \mathrm{C}$ contamination. These culture conditions are notably different from a similar species, Clostridium acetobutylicum, ${ }^{23}$ suggesting that a species-specific growth optimization stage is crucial for the efficient generation of uniformly labeled ${ }^{13} \mathrm{C}$ species.

To determine the glucose concentration supporting an optimally high culture density and the number of detectable metabolites, we investigated a range of glucose concentrations $(0.2-6 \% \mathrm{w} / \mathrm{v})$ (Figure $1 \mathrm{~b})$. The best ${ }^{13} \mathrm{C}$ yield/cost ratio was obtained with $4 \%$ glucose, and it provided a similar number of cells and $\mathrm{U}_{-}{ }^{13} \mathrm{C}$ intracellular metabolites compared to the use of $6 \%$ glucose (Figure $1 \mathrm{~b}$, Table $\mathrm{S} 1$ ). Consequently, $4 \% \mathrm{U}-{ }^{13} \mathrm{C}$ glucose, $\mathrm{CaCO}_{3}$, and biotin were used in the culture medium for the biosynthesis of $\mathrm{U}_{-}{ }^{13} \mathrm{C}$-IS from $C$. pasteurianum. This approach produced $\mathrm{U}_{-}{ }^{13} \mathrm{C}$-IS for the absolute quantification of 131 key intracellular metabolites of C. autoethanogenum and provided an increased metabolic coverage compared to similar methods. ${ }^{5,6,24-26}$ 

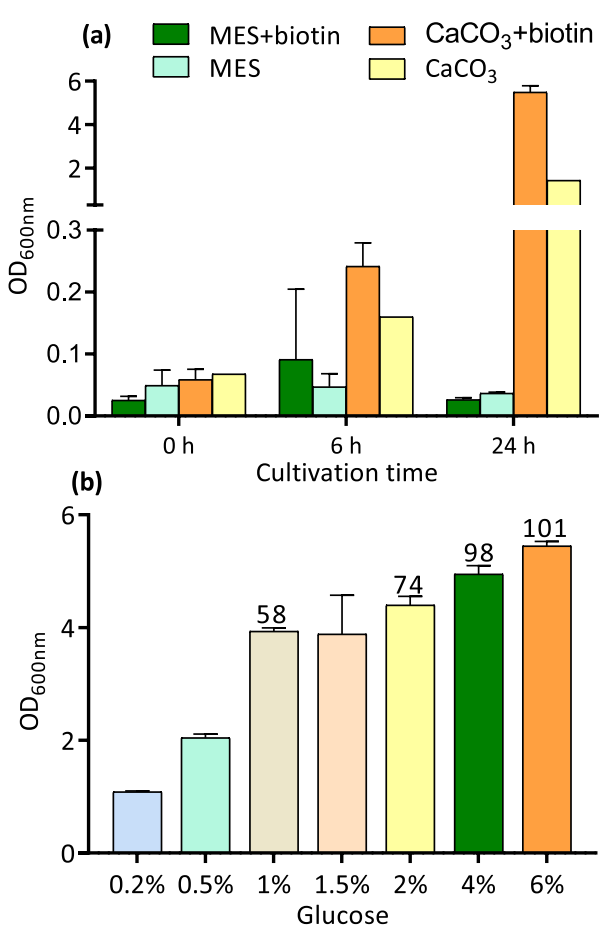

Figure 1. Optimization of the culture conditions for the optimum production of multiple $\mathrm{U}^{13} \mathrm{C}$-IS by $\mathrm{C}$. pasteurianum. The growth was monitored $\left(\mathrm{OD}_{600 \mathrm{~nm}}\right)$ at (a) 0, 6, and $24 \mathrm{~h}$ using $\mathrm{CaCO}_{3}$ and $\mathrm{MES}$ as culture buffers compared to the addition of biotin and (b) $24 \mathrm{~h}$ using $0.2-6 \%$ glucose as the carbon source; the number above the bar graph shows the number of the detected metabolites. The presence of biotin with $\mathrm{CaCO}_{3}$ provided the best growth compared to the rest, while $4 \%$ glucose was considered optimum.

LC-IDMS Validation and Confirmation of the Metabolite Purity from the $\mathrm{U}-{ }^{13} \mathrm{C}$ Extract. The quality and purity of the extracted $\mathrm{U}-{ }^{13} \mathrm{C}$ metabolites from the samples of C. pasteurianum grown on ${ }^{13} \mathrm{C}$ glucose were investigated for use as $\mathrm{U}_{-}{ }^{13} \mathrm{C}$-IS for LC-IDMS. The isotopic purity of the $\mathrm{U}^{13} \mathrm{C}$-IS in the pure extract of $\mathrm{U}^{13} \mathrm{C}$ C. pasteurianum $(n=3)$ showed that the majority of ${ }^{13} \mathrm{C}$ metabolites were $100 \%$ pure with a suitable signal-to-noise ratio $\geq 10$ (i.e., $\geq$ LOQ) to be used as $\mathrm{U}-{ }^{13} \mathrm{C}$ IS; however, few ${ }^{13} \mathrm{C}$ metabolites showed a minimal impurity of ${ }^{12} \mathrm{C}$ isotopes (Figure $\mathrm{S} 1$ ). Where, in a small number of cases, a ${ }^{12} \mathrm{C}$ endogenous metabolite was not matched by an individual ${ }^{13} \mathrm{C}$ IS metabolite in the mixture, the chemically most closely related ${ }^{13} \mathrm{C}$ IS metabolite was used to determine the ${ }^{12} \mathrm{C} /{ }^{13} \mathrm{C}$ ratio. The exact ${ }^{13} \mathrm{C}$ IS used for each endogenous metabolite is listed in Table S2. This indicates a good coverage of about $70 \%$ of C. autoethanogenum metabolites under investigation and more than twice the number of $\mathrm{U}_{-}{ }^{13} \mathrm{C}-\mathrm{IS}$ obtained previously with $\mathrm{U}_{-1}^{13} \mathrm{C}$ spirulina. ${ }^{6}$

The optimized LC-IDMS method demonstrated a complete resolution of all 131 metabolites in C. autoethanogenum (RT range: 4.00-12.40 min; typical chromatograms shown in Figure S2). Method validation (performed for each metabolite) demonstrated that the assay met the requirements for a quantitative LC-MS/MS method with respect to linearity, precision, accuracy, LOD, and LOQ (Table 1 summarizes data and Table S2 has full details). The detection of major central metabolic pathways, including tricarboxylic acid (TCA) pathway intermediates, nucleotides, amino acids, and $S$ adenosyl methionine cycle metabolites, was in many cases found to be improved, or at least equivalent, compared to previously reported LC-MS methods. ${ }^{27-34}$

Metabolic Profiling of C. autoethanogenum Bioreactors to Study the Effect of the Acidic pH Shift on the Production of High-Value Chemicals. The study involved an acidic $\mathrm{pH}$ shift of continuous cultures using $\mathrm{CO}$ as the sole single carbon substrate (Figure 2). At the initial condition of $15 \mathrm{~mL} / \mathrm{min} \mathrm{CO}$ inflow and $\mathrm{pH} 5.0$, the level of acetate was higher than that of ethanol and was observed until day 11 (acetogenesis, phase 1). This low flow rate was chosen to allow the culture to adapt to the setup, whereas the $\mathrm{pH}$ was found optimally for C. autoethanogenum growth on $\mathrm{C} 1$ gas. $^{32,33}$ Then, the $\mathrm{CO}$ flow rate was increased to $27 \mathrm{~mL} / \mathrm{min}$ to maximize acetate and ethanol formation (acetogenesis, phase 2). This $\mathrm{CO}$ shift increased the level of both ethanol and acetate at the same rate with further acetate accumulation. As the $\mathrm{pH}$ was reduced to 4.7 , acid production decreased with the increase in solvent (ethanol and 2,3-butanediol) production due to reassimilation of acids into solvents. ${ }^{34} \mathrm{~A}$ further drop in $\mathrm{pH}$ from 4.7 to 4.5 showed almost complete conversion of acetate to ethanol ${ }^{13}$ (solventogenesis, phase 3). In all three phases, the use of $\mathrm{CO}$ gas supplied was measured and was constant in each phase at $67-68 \%$.

LC-IDMS was applied to monitor the changes in the intracellular metabolic profiles of C. autoethanogenum linked to the production of ethanol, acetate, and 2,3-butanediol in a series of bioreactor experiments (Figure 2). The intracellular metabolites of $C$. autoethanogenum were successfully quantified using the LC-IDMS in the daily samples $(n=3)$ over 40 days; a heatmap of the measured metabolites over time is available in Table S3. The orderly progression of changes in the intracellular metabolite concentrations of $C$. autoethanogenum was observed during acetogenesis (CO 15 to $27 \mathrm{~mL} / \mathrm{min}$ ) and solventogenesis $(\mathrm{pH}$ 5.0-4.5). Figure S3 shows a direct comparison of a selected set of metabolites during each $\mathrm{CO}$ or $\mathrm{pH}$ changes. The change in the $\mathrm{CO}$ flow rate had a less significant effect compared to the $\mathrm{pH}$ shift, and the concentrations of most of the intracellular metabolites had significantly changed during the $\mathrm{pH}$ shift to 4.5 where $C$. autoethanogenum was mainly in the solventogenesis phase. For instance, the concentration of AMP during acetogenesis was relatively high and continued to increase during solventogenesis. This may indicate that ATP is heavily utilized during acetate reassimilation for ethanol production, as previously stated. $^{34}$ Increased levels of some amino acids, such as citrulline, homoserine, aspartate, proline, threonine, and arginine, were also observed during acetogenesis. Others, such as tryptophan, gluconate, inosine, $\mathrm{NAD}^{+}, \mathrm{NADP}^{+}$, pyruvate, and 2-oxoglutarate, were decreased. The increased level of arginine from acetogenesis to solventogenesis was similar to C. acetobutylicum. ${ }^{34}$ Lactate, which is of a biotechnological interest, and succinate from the branchedTCA pathway of C. autoethanogenum, were also increased, which is consistent with the results reported by Marcellin et al. $^{20}$

Robust Regression Analysis for the Estimation of Metabolite Concentrations at a Steady State. The average levels of metabolites during acetogenesis (phase 1 and phase 2) and solventogenesis (phase 3) were estimated using the optimized robust regression procedure to provide an accurate comparison between the different states due to $\mathrm{CO}$ and/or $\mathrm{pH}$ modulation. Figure 3 shows the minimized effect of the outliers in the determination of the concentrations of 
Table 1. Validation Summary of the LC-IDMS for the Quantification of 131 Bacterial Metabolites ${ }^{a}$

\begin{tabular}{|c|c|c|c|c|c|c|c|c|c|c|c|}
\hline \multicolumn{12}{|c|}{ A. Validation Summary } \\
\hline description & $\begin{array}{l}\text { number of } \\
\text { metabolites }\end{array}$ & \multicolumn{2}{|c|}{ description } & $\begin{array}{l}\text { number of } \\
\text { metabolites }\end{array}$ & descriptic & & $\begin{array}{l}\text { number of } \\
\text { metabolites }\end{array}$ & \multicolumn{3}{|c|}{ description } & $\begin{array}{l}\text { mber of } \\
\text { tabolites }\end{array}$ \\
\hline $\begin{array}{l}\text { quantification } \\
\text { (LC-IDMS) }\end{array}$ & 131 & \multicolumn{2}{|c|}{ linearity } & & precision & & & \multicolumn{3}{|c|}{ accuracy } & \\
\hline IS & & \multicolumn{2}{|c|}{$R^{2} \geq 0.99$} & 131 & low $(5 \mu \mathrm{M})$ & & & \multicolumn{3}{|c|}{ low $(5 \mu \mathrm{M})$} & \\
\hline $\mathrm{U}-{ }^{13} \mathrm{C}-\mathrm{IS}$ & 93 & \multicolumn{2}{|c|}{ LOD } & & RSD $<10 \%$ & & 109 & \multicolumn{2}{|c|}{$100 \pm 10 \%$} & \multicolumn{2}{|r|}{92} \\
\hline alternative U- ${ }^{13} \mathrm{C}$-IS & 38 & \multicolumn{2}{|c|}{$\leq 1 \mathrm{nM}$} & 17 & RSD $10-15 \%$ & & 22 & \multicolumn{2}{|c|}{$100 \pm 15 \%$} & & 131 \\
\hline linear range & & \multicolumn{2}{|c|}{$1-10 \mathrm{nM}$} & 59 & $\begin{array}{l}\text { medium } \\
(10-25 \mu \mathrm{M}\end{array}$ & & & \multicolumn{3}{|c|}{$\begin{array}{l}\text { medium } \\
\qquad(10-25 \mu \mathrm{M})\end{array}$} & \\
\hline $0.5-25 \mu \mathrm{M}$ & 6 & \multicolumn{2}{|c|}{$20-100 \mathrm{nM}$} & 38 & RSD $<10 \%$ & & 120 & \multicolumn{2}{|c|}{$100 \pm 10 \%$} & \multicolumn{2}{|r|}{105} \\
\hline $0.5-50 \mu \mathrm{M}$ & 40 & \multicolumn{2}{|c|}{$0.25-1 \mu \mathrm{M}$} & 17 & RSD 10-14\% & & 11 & \multicolumn{2}{|c|}{$100 \pm 15 \%$} & \multicolumn{2}{|r|}{131} \\
\hline $0.5-100 \mu \mathrm{M}$ & 67 & \multicolumn{2}{|c|}{ LOQ } & & high $(25-50$ & u) & & \multicolumn{3}{|c|}{ high $(25-50 \mu \mathrm{M})$} & \\
\hline $1-25 \mu \mathrm{M}$ & 2 & \multicolumn{2}{|c|}{$1 \mathrm{nM}$} & 8 & RSD $<10 \%$ & & 116 & \multicolumn{2}{|c|}{$100 \pm 7 \%$} & \multicolumn{2}{|r|}{131} \\
\hline $1-50 \mu \mathrm{M}$ & 3 & \multicolumn{2}{|c|}{$10-50 \mathrm{nM}$} & 62 & \multicolumn{2}{|l|}{ RSD $10-14 \%$} & 15 & & & & \\
\hline $1-100 \mu \mathrm{M}$ & 12 & \multicolumn{2}{|c|}{$0.1-1.0 \mu \mathrm{M}$} & 60 & & & & & & & \\
\hline $5-200 \mu \mathrm{M}$ & 1 & \multicolumn{2}{|c|}{$5 \mu \mathrm{M}$} & 1 & & & & & & & \\
\hline & & & B. Valida & ation Summary B & ased on Metab & lite Class & & & & & \\
\hline & & & & & & & ecision $^{b}(\%)$ & & & ccuracy ${ }^{c}(\%$ & \\
\hline class & $\begin{array}{l}\text { number of } \\
\text { metabolites }\end{array}$ & $\begin{array}{l}\text { LOD } \\
(\mathrm{nM})\end{array}$ & LOQ $(\mu \mathrm{M})$ & $R^{2}$ & $\begin{array}{l}\text { linear } \\
\text { range } \\
(\mu \mathrm{M})\end{array}$ & low $(\%)$ & $\begin{array}{l}\text { medium } \\
(\%)\end{array}$ & $\begin{array}{l}\text { high } \\
(\%)\end{array}$ & low (\%) & $\begin{array}{l}\text { medium } \\
(\%)\end{array}$ & high (\%) \\
\hline amino acid derivatives & 8 & $1-100$ & $0.01-0.25$ & $0.987-0.997$ & $0.5-100$ & $1-15$ & $2-10$ & $2-10$ & $85-111$ & $94-107$ & $98-103$ \\
\hline amino acids & 23 & $<1-500$ & $0.001-5.00$ & $0.985-0.997$ & $0.5-100$ & $1-13$ & $1-14$ & $1-13$ & $85-111$ & $85-114$ & $96-107$ \\
\hline $\mathrm{CoA} / \mathrm{CoA}$ derivatives & 7 & $10-500$ & $0.10-1.00$ & $0.986-0.994$ & $0.5-100$ & $3-12$ & $1-10$ & $3-10$ & $85-115$ & $94-112$ & $97-103$ \\
\hline $\begin{array}{l}\text { mono-/disaccharides and } \\
\text { derivatives }\end{array}$ & 2 & $10-100$ & $0.05-0.25$ & 0.994 & $0.5-100$ & $1-3$ & $3-4$ & 6 & $87-95$ & $97-112$ & $98-101$ \\
\hline organic acids & 35 & $<1-500$ & $0.001-1.00$ & $0.985-0.996$ & $0.5-100$ & $1-15$ & $1-11$ & $1-14$ & $89-115$ & $86-113$ & $96-105$ \\
\hline phosphorylated derivatives & 5 & $10-500$ & $0.05-1.00$ & $0.985-0.995$ & $0.5-100$ & $3-15$ & $1-9$ & $3-7$ & $85-94$ & $92-107$ & $98-106$ \\
\hline $\begin{array}{l}\text { phosphorylated } \\
\text { mono-/disaccharides } \\
\text { and derivatives }\end{array}$ & 8 & $<1-250$ & $0.001-1.00$ & $0.986-0.993$ & $0.5-100$ & $2-13$ & $1-11$ & $2-11$ & $85-113$ & $92-114$ & $96-105$ \\
\hline $\begin{array}{l}\text { phosphorylated purines } \\
\text { and purine derivatives }\end{array}$ & 16 & $<1-500$ & $0.001-0.50$ & $0.987-0.997$ & $0.5-100$ & $1-13$ & $1-8$ & $1-9$ & $85-113$ & $89-114$ & $95-104$ \\
\hline $\begin{array}{l}\text { phosphorylated } \\
\text { pyrimidines and } \\
\text { pyrimidine derivatives }\end{array}$ & 12 & $<1-250$ & $0.001-0.50$ & $0.987-0.997$ & $0.5-100$ & $2-6$ & $2-9$ & $3-12$ & $85-112$ & $86-113$ & $97-105$ \\
\hline $\begin{array}{l}\text { purines and purine } \\
\text { derivatives }\end{array}$ & 9 & $<1-100$ & $0.001-0.25$ & $0.985-0.994$ & $0.5-100$ & $0.4-15$ & $2-13$ & $1-9$ & $85-107$ & $88-107$ & $98-105$ \\
\hline $\begin{array}{l}\text { pyrimidines and } \\
\text { pyrimidine derivatives }\end{array}$ & 6 & $<1-250$ & $0.001-1.00$ & $0.986-0.995$ & $0.5-100$ & $2-13$ & $2-12$ & $3-9$ & $86-108$ & $101-110$ & $97-103$ \\
\hline
\end{tabular}

${ }^{a}$ Full details of the method validation of all analytes are given in the Supporting Information for publication, Table S2. ${ }^{b}$ Precisions were calculated at low: $5 \mu \mathrm{M}$, medium: $25 \mu \mathrm{M}$, and high: $100 \mu \mathrm{M}$ except for those with an upper limit of quantification (ULOQ) less than $100 \mu \mathrm{M}$, which were measured at low $=5 \mu \mathrm{M}$, medium $=25 \mu \mathrm{M}$, and high $=50 \mu \mathrm{M}$ for ULOQ of $50 \mu \mathrm{M}$ and Low $=5 \mu \mathrm{M}$, medium $=10 \mu \mathrm{M}$, and high $=25 \mu \mathrm{M}$ for ULOQ of $25 \mu \mathrm{M}$, abbreviations: LOD: limit of detection, LOQ: limit of quantification, and $\mathrm{U}-{ }^{13} \mathrm{C}$-IS: uniformly labeled ${ }^{13} \mathrm{C}$-IS. ${ }^{c}$ Accuracy was calculated at low: $5 \mu \mathrm{M}$, medium: $25 \mu \mathrm{M}$, and high: $100 \mu \mathrm{M}$ except for those with an ULOQ less than $100 \mu \mathrm{M}$, which were measured at low $=5$ $\mu \mathrm{M}$, medium $=25 \mu \mathrm{M}$, and high $=50 \mu \mathrm{M}$ for ULOQ of $50 \mu \mathrm{M}$ and low $=5 \mu \mathrm{M}$, medium $=10 \mu \mathrm{M}$, and high $=25 \mu \mathrm{M}$ for ULOQ of $25 \mu \mathrm{M}$.

pyruvate, as an example, across the different growth phases of C. autoethanogenum using robust regression compared to standard averaging (a selected set of metabolites are also presented in Figure S4-S6). Such improvement in the quality of the metabolic data is important for a reliable representation of microbial metabolism since more and higher constrained experimental data restrict the variability of internal model variables. Therefore, models and their inherent assumptions about the network structure, mechanisms, and regulation can be validated more accurately and changed when deemed necessary. Consequently, the improved quality of the metabolic data allows for the improvement of metabolic models covering a wide range of conditions without losing proper biological internal states. Significantly changed metabolites between every two steady states were identified using $p$ values generated with a two-sample KolmogorovSmirnov test at a $95 \%$ confidence level (for full details, see
Table S4). Robust regression reduced the impact of outliers on the estimation of modeling parameters by an automated assignment of weights to the data points. In contrast, in common calculation of averages, all data points have the same weight and, hence, outliers can significantly reduce the accuracy and precision of the estimates. Consequently, robust regression generates estimates closer to the real intracellular concentration and less uncertainty than normal averaging. ${ }^{21,22}$

Metabolic Pathway Analysis and Mapping. Pathway analysis of the average metabolite levels during acetogenic and solventogenic steady states (Figure S7) was performed using MetaboAnalyst, ${ }^{35}$ and the affected pathways are listed in Table S5. Metabolic pathway analysis showed that reactions of the branched TCA pathway and alanine, aspartate, and glutamate metabolism were the most affected pathways associated with the $\mathrm{pH}$ shift in the bioreactor of $C$. autoethanogenum. In $C$. autoethanogenum, similar to C. acetobutylicum and Clostridium 


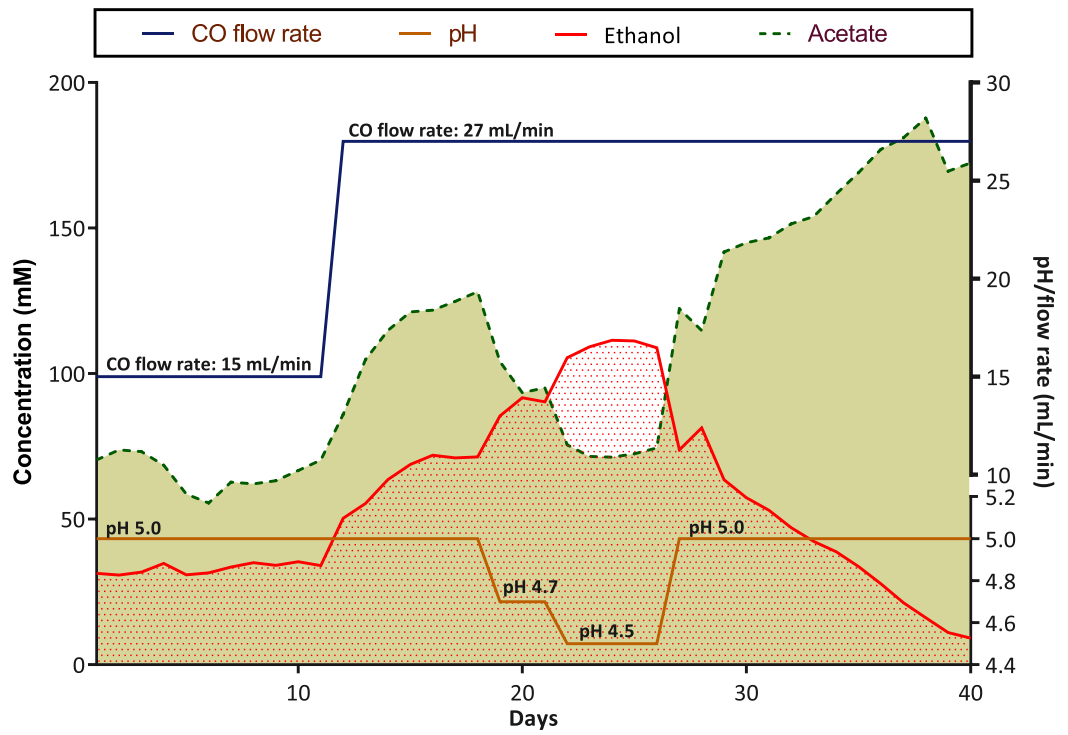

Figure 2. Production of ethanol and acetate and the effect of the $\mathrm{pH}$ shift on the conversion of acetate to ethanol by $\mathrm{C}$. autoethanogenum. At the initial condition of $15 \mathrm{~mL} / \mathrm{min} \mathrm{CO}$ flow and $\mathrm{pH} \mathrm{5}$, the level of acetate was higher than that of ethanol (acetogenesis). The CO flow rate was increased to $27 \mathrm{~mL} / \mathrm{min}$, in which the level of both ethanol and acetate increased. As the $\mathrm{pH}$ was reduced to 4.7 , acid production decreased with an increase in ethanol production (solventogenic phase). A further drop in $\mathrm{pH}$ to 4.5 showed the complete conversion of acetate to ethanol.

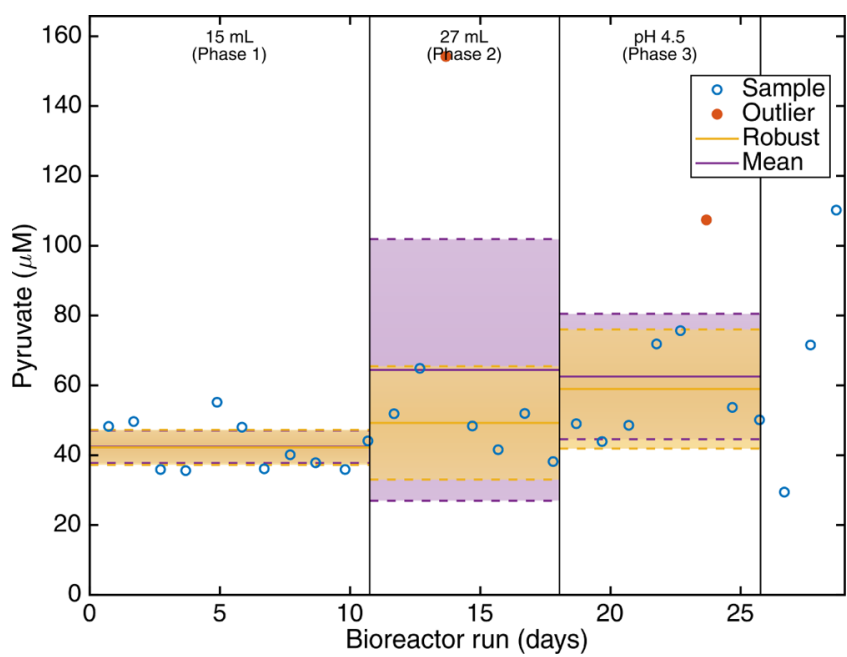

Figure 3. Determination of the pyruvate steady-state concentrations across the different growth phases of $C$. autoethanogenum using robust regression compared to standard average. It shows the minimized effect of the outliers, such as the ones shown on phases 2 and 3 (top) in the determination of the steady-state concentrations of pyruvate using robust regression. The robust regression line (yellow) fits within the sample range in phase 2 compared to the standard mean line (violet), hence providing a robust estimate of the steady-state concentration.

liungdahlii, ${ }^{36}$ there is an evidence that the TCA pathway is bifurcated ${ }^{37}$ and the oxidative branch of the pentose phosphate pathway is absent, ${ }^{38}$ making it difficult to define the enzymatic activity in these pathways. However, the level of malate, aspartate, and glutamate was found to be high during solventogenesis in C. autoethanogenum. ${ }^{34}$ This may indicate a similar enzymatic activity to $C$. acetobutylicum, in which the electron-consuming branch of the TCA pathway was found shut down during solventogenesis, but the oxidative part of the TCA pathway was active and responsible for the accumulation of aspartate and glutamate, consistent with the results obtained here. ${ }^{34}$ The average metabolite levels were also mapped using
Escher biological pathway maps ${ }^{39}$ based on a recent $C$. autoethanogenum genome model. ${ }^{40}$ Mapping the metabolite data on the metabolic pathways and reactions of $C$. autoethanogenum improved our understanding of the metabolic changes associated with $\mathrm{CO}$ and/or $\mathrm{pH}$ modulation (Figure 4). For example, the concentrations of pyruvate and acetyl CoA were relatively high during solventogenesis compared to acetogenesis, while the acetyl phosphate level was decreased. The accumulation of pyruvate and acetyl-CoA may play an indirect role in the production of ethanol from acetate and acetaldehyde. The increased level of ethanol associated with the decreased level of acetate during $\mathrm{pH}$ reduction was as a result of ferredoxin-dependent acetaldehyde oxidoreductase $(\mathrm{AFOR})^{41}$ and alcohol dehydrogenase $(\mathrm{ADH})$, the enzymes responsible for the conversion of acetate to ethanol, ${ }^{20}$ which was consistent with a similar microorganism. ${ }^{42}$

\section{CONCLUSIONS}

We have successfully developed and validated a new LCIDMS method for the analysis of 131 intracellular metabolites using C. pasteurianum as a source of ${ }^{13} \mathrm{C}$-labeled IS. Our method was applied here to the analysis of a series of bioreactor samples, providing consistent and valuable data for the quantitative modeling of metabolic pathways. Subsequently, we have used the method routinely for the analysis of many thousands of samples from other bioreactor studies on C. autoethanogenum. The developed LC-IDMS method demonstrated an improved throughput for the absolute quantification of the intracellular metabolites and has advantages for studies involving large sample sets where comprehensive coverage of intracellular pathways is required. In addition, the use of robust regression with the LC-IDMS data provides a powerful tool for the accurate estimation of the metabolite levels with greater resistance to outliers than standard regression/averaging. We believe that the combined method is suitable for the study of metabolic alterations caused by external changes and deliberate mutations in Clostridium sp. and will provide a high-quality input for computation 


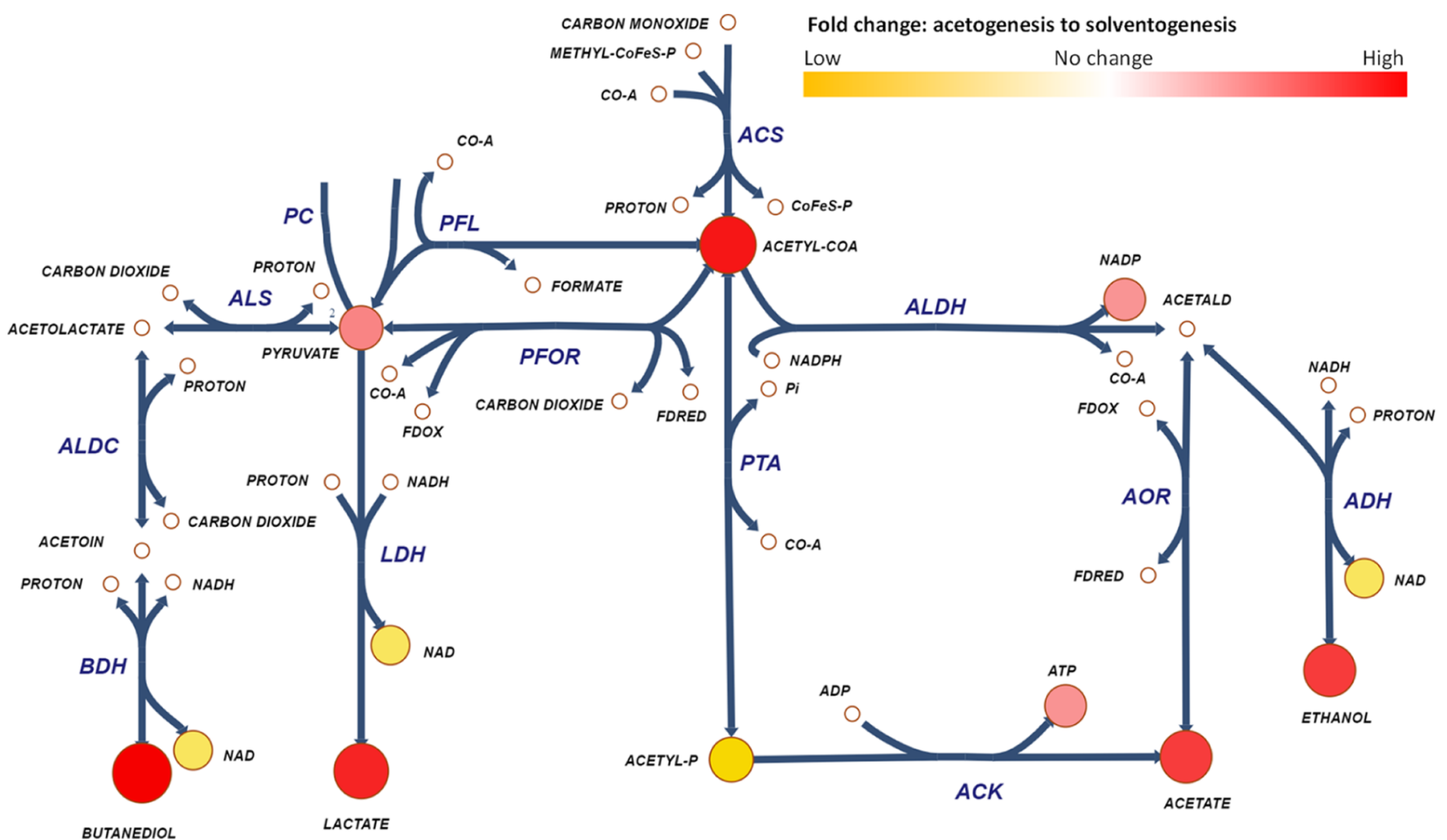

Figure 4. Mapping of the intracellular metabolites of $C$. autoethanogenum on its one-carbon metabolic pathway during the shift from acetogenesis to solventogenesis due to $\mathrm{pH}$ modulation. Circle size indicates the metabolite concentrations, small (low) $\rightarrow$ large (high), while small plain circles represent unchanged/unmapped metabolites. ACK: acetate kinase, ACS: acetyl CoA synthetase, ADH: alcohol dehydrogenase, ALDC: acetolactate decarboxylase, ALDH: acetaldehyde dehydrogenase, ALS: acetolactate synthase, AOR: acetaldehyde oxidoreductase, BDH: $(R, R)$-butanediol dehydrogenase, LDH: Lactate dehydrogenase, PC: pyruvate-carboxylase, PFL: pyruvate formate lyase, PFOR: pyruvate ferredoxin oxidoreductase, and PTA: phosphate acetyltransferase.

modeling. The biosynthesized ${ }^{13} \mathrm{C}$ LC-IDMS approach has the potential to be adapted to other types of microorganisms.

\section{ASSOCIATED CONTENT}

\section{SI Supporting Information}

The Supporting Information is available free of charge at https://pubs.acs.org/doi/10.1021/acsomega.0c05588.

LC-IDMS analysis, culture media for C. pasteurianum, purity of the $\mathrm{U}_{-1}^{13} \mathrm{C}$-IS in the extract of C. pasteurianum grown on $\mathrm{U}^{13} \mathrm{C}$ glucose, extracted ion chromatograms of a selected set of the key metabolites of $C$. autoethanogenum, direct comparison of the level of a selected set of metabolites measured at different $\mathrm{CO}$ or $\mathrm{pH}$ changes in the bioreactor samples of C. autoethanogenum, determination of the steady-state concentrations of a selected set of metabolites across the different growth phases of $C$. autoethanogenum using robust regression compared to standard average, and measured level of $C$. autoethanogenum metabolites in acetogenesis compared to solventogenesis (PDF)

Coverage of the key selected metabolites of $C$. autoethanogenum by $C$. pasteurianum, validation of the developed LC-IDMS, measured intracellular metabolites of $C$. autoethanogenum throughout the CO-fed bioreactor run, average concentrations of $C$. autoethanogenum metabolites at different steady states, and pathway analysis of the measured intracellular metabolites of $C$. autoethanogenum (XLSX)

\section{AUTHOR INFORMATION}

\section{Corresponding Author}

David A. Barrett - Centre for Analytical Bioscience, Advanced Materials and Healthcare Technologies Division, School of Pharmacy, University of Nottingham, Nottingham NG7

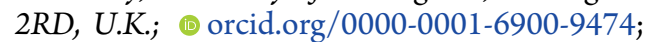
Phone: +44(0)115 9515062; Email: David.Barrett@ nottingham.ac.uk

\section{Authors}

Laudina Safo - Centre for Analytical Bioscience, Advanced Materials and Healthcare Technologies Division, School of Pharmacy, University of Nottingham, Nottingham NG7 2RD, U.K.

Salah Abdelrazig - Centre for Analytical Bioscience, Advanced Materials and Healthcare Technologies Division, School of Pharmacy, University of Nottingham, Nottingham NG7 2RD, U.K.; (1) orcid.org/0000-0001-6231-1267

Alexander Grosse-Honebrink - Biodiscovery Institute, School of Chemistry, University of Nottingham, Nottingham NG7 2RD, U.K.

Thomas Millat - Clostridia Research Group, BBSRC/EPSCR Synthetic Biology Research Centre (SBRC), Biodiscovery Institute, School of Life Sciences, University of Nottingham, Nottingham NG7 2RD, U.K.

Anne M. Henstra - Clostridia Research Group, BBSRC/ EPSCR Synthetic Biology Research Centre (SBRC), Biodiscovery Institute, School of Life Sciences, University of Nottingham, Nottingham NG7 2RD, U.K. 
Rupert Norman - Clostridia Research Group, BBSRC/ EPSCR Synthetic Biology Research Centre (SBRC), Biodiscovery Institute, School of Life Sciences, University of Nottingham, Nottingham NG7 2RD, U.K.

Neil R. Thomas - Biodiscovery Institute, School of Chemistry, University of Nottingham, Nottingham NG7 2RD, U.K.

Klaus Winzer - Clostridia Research Group, BBSRC/EPSCR Synthetic Biology Research Centre (SBRC), Biodiscovery Institute, School of Life Sciences, University of Nottingham, Nottingham NG7 2RD, U.K.

Nigel P. Minton - Clostridia Research Group, BBSRC/ EPSCR Synthetic Biology Research Centre (SBRC), Biodiscovery Institute, School of Life Sciences, University of Nottingham, Nottingham NG7 2RD, U.K.; 이이.org/ 0000-0002-9277-1261

Dong-Hyun Kim - Centre for Analytical Bioscience, Advanced Materials and Healthcare Technologies Division, School of Pharmacy, University of Nottingham, Nottingham NG7 2RD, U.K.; (1) orcid.org/0000-0002-3689-2130

Complete contact information is available at:

https://pubs.acs.org/10.1021/acsomega.0c05588

\section{Author Contributions}

"L.S. and S.A. are joint first authors.

\section{Notes}

The authors declare no competing financial interest.

\section{ACKNOWLEDGMENTS}

This work was supported by the UK Biotechnology and Biological Sciences Research Council (BBSRC) [grant numbers BB/K00283X/1 and BB/L013940/1].

\section{REFERENCES}

(1) Sumner, L. W.; Mendes, P.; Dixon, R. A. Plant metabolomics: large-scale phytochemistry in the functional genomics era. Phytochemistry 2003, 62, 817-836.

(2) Nielsen, J.; Oliver, S. The next wave in metabolome analysis. Trends Biotechnol. 2005, 23, 544-546.

(3) Sargent, M.; Harte, R.; Harrington, C. Guidelines for Achieving High Accuracy in Isotope Dilution Mass Spectrometry (IDMS); RSC, 2002; p 1-34.

(4) Zhou, J.; Liu, H.; Liu, Y.; Liu, J.; Zhao, X.; Yin, Y. Development and Evaluation of a Parallel Reaction Monitoring Strategy for LargeScale Targeted Metabolomics Quantification. Anal. Chem. 2016, 88, 4478-4486.

(5) Kim, D.-H.; Achcar, F.; Breitling, R.; Burgess, K. E.; Barrett, M. P. LC-MS-based absolute metabolite quantification: application to metabolic flux measurement in trypanosomes. Metabolomics 2015, 11, $1721-1732$.

(6) Schatschneider, S.; Abdelrazig, S.; Safo, L.; Henstra, A. M.; Millat, T.; Kim, D.-H.; Winzer, K.; Minton, N. P.; Barrett, D. A. Quantitative Isotope-Dilution High-Resolution-Mass-Spectrometry Analysis of Multiple Intracellular Metabolites in Clostridium autoethanogenum with Uniformly 13C-Labeled Standards Derived from Spirulina. Anal. Chem. 2018, 90, 4470-4477.

(7) Hermann, G.; Schwaiger, M.; Volejnik, P.; Koellensperger, G. 13C-labelled yeast as internal standard for LC-MS/MS and LC high resolution MS based amino acid quantification in human plasma. $J$. Pharm. Biomed. Anal. 2018, 155, 329-334.

(8) Köpke, M.; Mihalcea, C.; Liew, F.; Tizard, J. H.; Ali, M. S.; Conolly, J. J.; Al-Sinawi, B.; Simpson, S. D. 2,3-Butanediol Production by Acetogenic Bacteria, an Alternative Route to Chemical Synthesis, Using Industrial Waste Gas. Appl. Environ. Microbiol. 2011, 77, 54675475.
(9) Abrini, J.; Naveau, H.; Nyns, E.-J. Clostridium autoethanogenum, sp. nov., an anaerobic bacterium that produces ethanol from carbon monoxide. Arch. Microbiol. 1994, 161, 345-351.

(10) Köpke, M.; Gerth, M. L.; Maddock, D. J.; Mueller, A. P.; Liew, F.; Simpson, S. D.; Patrick, W. M. Reconstruction of an acetogenic 2,3-butanediol pathway involving a novel NADPH-dependent primary-secondary alcohol dehydrogenase. Appl. Environ. Microbiol. 2014, 80, 3394.

(11) Abubackar, H. N.; Veiga, M. C.; Kennes, C. Biological conversion of carbon monoxide to ethanol: Effect of $\mathrm{pH}$, gas pressure, reducing agent and yeast extract. Bioresour. Technol. 2012, 114, 518522.

(12) Abubackar, H. N.; Bengelsdorf, F. R.; Dürre, P.; Veiga, M. C.; Kennes, C. Improved operating strategy for continuous fermentation of carbon monoxide to fuel-ethanol by clostridia. Appl. Energy 2016, 169, 210-217.

(13) Abubackar, H. N.; Fernández-Naveira, Á.; Veiga, M. C.; Kennes, C. Impact of cyclic $\mathrm{pH}$ shifts on carbon monoxide fermentation to ethanol by Clostridium autoethanogenum. Fuel 2016, 178, 56-62.

(14) Carey, J.; Nguyen, T.; Korchak, J.; Beecher, C.; de Jong, F.; Lane, A. L. An Isotopic Ratio Outlier Analysis Approach for Global Metabolomics of Biosynthetically Talented Actinomycetes. Metabolites 2019, 9, 181.

(15) de Jong, F. A.; Beecher, C. Addressing the current bottlenecks of metabolomics: Isotopic Ratio Outlier Analysis ${ }^{\mathrm{TM}}$, an isotopiclabeling technique for accurate biochemical profiling. Bioanalysis 2012, 4, 2303-2314.

(16) Simillion, C.; Semmo, N.; Idle, J.; Beyoğlu, D. Robust Regression Analysis of GCMS Data Reveals Differential Rewiring of Metabolic Networks in Hepatitis B and C Patients. Metabolites 2017, $7,51$.

(17) Schwarz, K. M.; Grosse-Honebrink, A.; Derecka, K.; Rotta, C.; Zhang, Y.; Minton, N. P. Towards improved butanol production through targeted genetic modification of Clostridium pasteurianum. Metab. Eng. 2017, 40, 124-137.

(18) Biebl, H. Fermentation of glycerol by Clostridium pasteurianum - batch and continuous culture studies. J. Ind. Microbiol. Biotechnol. 2001, 27, 18-26.

(19) FDA, FDA Guidance for Industry. Bioanalytical Method Validation; Food and Drug Administration, Centre for Drug Evaluation and Research (CDER), 2013.

(20) Marcellin, E.; Behrendorff, J. B.; Nagaraju, S.; DeTissera, S.; Segovia, S.; Palfreyman, R. W.; Daniell, J.; Licona-Cassani, C.; Quek, L.-e.; Speight, R.; Hodson, M. P.; Simpson, S. D.; Mitchell, W. P.; Köpke, M.; Nielsen, L. K. Low carbon fuels and commodity chemicals from waste gases - systematic approach to understand energy metabolism in a model acetogen. Green Chem. 2016, 18, 3020-3028.

(21) Holland, P. W.; Welsch, R. E. Robust regression using iteratively reweighted least-squares. Commun. Stat. Theor. Methods 1977, 6, 813-827.

(22) Street, J. O.; Carroll, R. J.; Ruppert, D. A Note on Computing Robust Regression Estimates Via Iteratively Reweighted Least Squares. Am. Statistician 1988, 42, 152-154.

(23) Lehmann, D.; Lütke-Eversloh, T. Switching Clostridium acetobutylicum to an ethanol producer by disruption of the butyrate/butanol fermentative pathway. Metab. Eng. 2011, 13, 464473.

(24) Berg, T.; Strand, D. H. C-13 labelled internal standards-A solution to minimize ion suppression effects in liquid chromatography-tandem mass spectrometry analyses of drugs in biological samples? J. Chromatogr. A 2011, 1218, 9366-9374.

(25) Cui, J.; Zhang, J.; Zhu, X.; Bai, F.; Feng, Y.; Guan, W.; Cui, Q. Separation and Quantification of Water-Soluble Cellular Metabolites in Clostridium thermocellum using Liquid Chromatography-Isotope Dilution Tandem Mass Spectrometry. Anal. Lett. 2013, 46, 27672786. 
(26) Lu, W.; Kwon, Y. K.; Rabinowitz, J. D. Isotope Ratio-Based Profiling of Microbial Folates. J. Am. Soc. Mass Spectrom. 2007, 18, 898-909.

(27) Huang, Y.; Tian, Y.; Zhang, Z.; Peng, C. A HILIC-MS/MS method for the simultaneous determination of seven organic acids in rat urine as biomarkers of exposure to realgar. J. Chromatogr. B: Anal. Technol. Biomed. Life Sci. 2012, 905, 37-42.

(28) Lu, W.; Kimball, E.; Rabinowitz, J. D. A High-Performance Liquid Chromatography-Tandem Mass Spectrometry Method for Quantitation of Nitrogen-Containing Intracellular Metabolites. J. Am. Soc. Mass Spectrom. 2006, 17, 37-50.

(29) Luo, B.; Groenke, K.; Takors, R.; Wandrey, C.; Oldiges, M. Simultaneous determination of multiple intracellular metabolites in glycolysis, pentose phosphate pathway and tricarboxylic acid cycle by liquid chromatography-mass spectrometry. J. Chromatogr. A 2007, 1147, 153-164.

(30) Cordell, R. L.; Hill, S. J.; Ortori, C. A.; Barrett, D. A. Quantitative profiling of nucleotides and related phosphate-containing metabolites in cultured mammalian cells by liquid chromatography tandem electrospray mass spectrometry. J. Chromatogr. B: Anal. Technol. Biomed. Life Sci. 2008, 871, 115-124.

(31) Bajad, S. U.; Lu, W.; Kimball, E. H.; Yuan, J.; Peterson, C.; Rabinowitz, J. D. Separation and quantitation of water soluble cellular metabolites by hydrophilic interaction chromatography-tandem mass spectrometry. J. Chromatogr. A 2006, 1125, 76-88.

(32) Klasson, K. T.; Ackerson, M. D.; Clausen, E. C.; Gaddy, J. L. Bioconversion of synthesis gas into liquid or gaseous fuels. Enzyme Microb. Technol. 1992, 14, 602-608.

(33) Cotter, J. L.; Chinn, M. S.; Grunden, A. M. Ethanol and acetate production by Clostridium ljungdahlii and Clostridium autoethanogenum using resting cells. Bioprocess Biosyst. Eng. 2009, 32, 369-380.

(34) Amador-Noguez, D.; Brasg, I. A.; Feng, X.-J.; Roquet, N.; Rabinowitz, J. D. Metabolome Remodeling during the AcidogenicSolventogenic Transition in Clostridium acetobutylicum. Appl. Environ. Microbiol. 2011, 77, 7984-7997.

(35) Chong, J.; Xia, J. MetaboAnalystR: an R package for flexible and reproducible analysis of metabolomics data. Bioinformatics 2018, 34, 4313-4314.

(36) Köpke, M.; Held, C.; Hujer, S.; Liesegang, H.; Wiezer, A.; Wollherr, A.; Ehrenreich, A.; Liebl, W.; Gottschalk, G.; Dürre, P. Clostridium ljungdahlii represents a microbial production platform based on syngas. Proc. Natl. Acad. Sci. U.S.A. 2010, 107, 13087.

(37) Amador-Noguez, D.; Feng, X.-J.; Fan, J.; Roquet, N.; Rabitz, H.; Rabinowitz, J. D. Systems-Level Metabolic Flux Profiling Elucidates a Complete, Bifurcated Tricarboxylic Acid Cycle in Clostridium acetobutylicum. J. Bacteriol. 2010, 192, 4452-4461.

(38) Crown, S. B.; Indurthi, D. C.; Ahn, W. S.; Choi, J.; Papoutsakis, E. T.; Antoniewicz, M. R. Resolving the TCA cycle and pentosephosphate pathway of Clostridium acetobutylicum ATCC 824: Isotopomer analysis, in vitro activities and expression analysis. Biotechnol. J. 2011, 6, 300-305.

(39) King, Z. A.; Dräger, A.; Ebrahim, A.; Sonnenschein, N.; Lewis, N. E.; Palsson, B. O.; Escher. A Web Application for Building, Sharing, and Embedding Data-Rich Visualizations of Biological Pathways. PLoS Comput. Biol. 2015, 11, No. e1004321.

(40) Norman, R. O.; Millat, T.; Schatschneider, S.; Henstra, A.; Breitkopf, R.; Pander, B.; Annan, F.; Piatek, P.; Hartman, H.; Poolman, M.; Fell, D.; Winzer, K.; Minton, N.; Hodgman, C. A genome-scale model of Clostridium autoethanogenum reveals optimal bioprocess conditions for high-value chemical production from carbon monoxide. Eng. Biol. 2019, 3, 32.

(41) Ohashi, Y.; Hirayama, A.; Ishikawa, T.; Nakamura, S.; Shimizu, K.; Ueno, Y.; Tomita, M.; Soga, T. Depiction of metabolome changes in histidine-starved Escherichia coli by CE-TOFMS. Mol. BioSyst. 2008, 4, 135-147.

(42) Fernández-Naveira, Á.; Veiga, M. C.; Kennes, C. Effect of salinity on C1-gas fermentation by Clostridium carboxidivorans producing acids and alcohols. Amb. Express 2019, 9, 110. 